\title{
Continuously Varying Coupled Transmission Lines Applied to Design Band-Pass Filters
}

\author{
Marc Le Roy ${ }^{1}$, André Pérennec ${ }^{2}$, Serge Toutain ${ }^{3}$, Léon Claude Calvez ${ }^{2}$ \\ ${ }^{1}$ LEMI - IUT - Université de Rouen - 76821 Mont Saint Aignan, France \\ ${ }^{2}$ LEST (FRE CNRS n ${ }^{\circ}$. 2269), Université de Bretagne Occidentale, U.F.R. Sciences, BP 809, \\ 29285 Brest cedex, France, ENST de Bretagne, 29285 Brest cedex, France. \\ ${ }^{3}$ SEI, IRESTE, EP CNRS 63, rue C. Pauc, La chantrerie, BP 60601, 44306 Nantes cedex 3,
} France.

\begin{abstract}
This paper presents a technique to analyze and optimize Continuously Varying Transmission Lines (CVTL's), used to design microwave band-pass filters in planar technology. For millimeters waves, radiation losses can increase quickly, especially for sharp discontinuities in planar circuits. In this approach, rather than taking account of this effect in a full-wave analysis, we generate structures without discontinuities. Moreover, the line shape is optimized to reduce spurious responses out of the band-pass. For several filters, measurements are compared with simulation results.
\end{abstract}

Keywords: Nonuniform Transmission Lines, band-pass filters, optimization

\section{INTRODUCTION}

It has been shown [1] that for a simple stub, radiation increases dramatically beyond $140 \mathrm{GHz}$. When an electromagnetic simulation of a total mm-wave circuit is carried out, all these radiation losses are accounted for. However, computational requirements are very expensive in CPU time, and no optimization is conceivable. In a circuit, there are many sources of losses (conductor and dielectric, surface roughness). Our technique concerns the minimization of the radiation losses that appears for sharp discontinuities in planar filters. In previous papers [2,3], we have applied the Continuously Varying Transmission Lines (CVTL) method for low-pass filters carried out in coplanar technology. This technique is based on a simple formalism and no approximations are made in the successive steps. Moreover, 
optimization of the global shape is permitted. Very good agreement in frequencies were obtained up to $40 \mathrm{GHz}$.

Although there are many topologies for band-pass filters, only few papers have been reported on nonuniform coupled transmission lines. This paper introduces an adaptation of the CVTL's technique to coupled lines, one of the basic structure for band-pass filters. This approach is adapted to the design of band-pass filters through the inclusion of the corresponding constraints into the model. In comparison to the usual techniques, our method brings additional degrees of freedom which are used to control the out-of-band spurious responses of coupled band-pass filters.

\section{ANALYSIS METHOD FOR A SINGLE LINE : LOW-PASS FILTERS}

\subsection{Description of the line shape}

Until now in the literature, the only studied nonuniform transmission lines were those which are described by a mathematical simple form, such as linear, exponential, parabolic, cosine-squared and hyperbolic. But there were no methods for arbitrary line shape. Initially, the problem was to find a mathematical form for the continuous shape which permits the resolution of the telegrapher's equation. Moreover, an optimization process must be performed on the whole line to satisfy the filters specifications, for example. The cubic spline interpolation was chosen to describe the line shape. It allows to create a curve passing through a set of discrete points that minimize the ripples. It also ensures the continuity of the first and second derivatives. The impedance variation between two discrete points is described by the following cubic polynomial :

$$
Z(x)=Z_{0}+Z_{1} x+Z_{2} x^{2}+Z_{3} x^{3}
$$

with 


$$
\begin{aligned}
& Z_{0}=Z_{j} \\
& Z_{1}=-\frac{\ell}{3} Z^{\prime \prime}{ }_{j}-\frac{\ell}{6} Z^{\prime \prime}{ }_{j+1}-\frac{Z_{j}-Z_{j+1}}{\ell} \\
& Z_{2}=\frac{Z^{\prime \prime}{ }_{j}}{2} \\
& Z_{3}=\frac{-Z^{\prime \prime}{ }_{j}+Z^{\prime \prime}{ }_{j+1}}{6 \ell}
\end{aligned}
$$

where $x$ varies from 0 to $\ell . \ell$ is the line length between $Z_{j}$ and $Z_{j+1}$ which represent the ordinate of the $j^{\text {th }}$ and $(j+1)^{\text {th }}$ discrete points used to define the cubic spline interpolation. $Z{ }^{\prime \prime}{ }_{j}$ and $Z{ }^{\prime}{ }_{j+1}$ are the second derivative of the impedance variation $Z(x)$ at these respective points. Here, a lossless nonuniform line is considered. So, this relation can also be expressed in the form of :

$$
Z(x)=\sqrt{\frac{L(x)}{C(x)}}
$$

with $L(x)=\sqrt{L_{0} \cdot C_{0}} \cdot\left(Z_{0}+Z_{1} \cdot x+Z_{2} \cdot x^{2}+Z_{3} \cdot x^{3}\right)$

and

$$
C(x)=\frac{\sqrt{L_{0} \cdot C_{0}}}{Z_{0}+Z_{1} \cdot x+Z_{2} \cdot x^{2}+Z_{3} \cdot x^{3}}
$$

$Z_{0}$ is equal to $\sqrt{L_{0} / C_{0}}$ at the input end of the nonuniform transmission line, where $L_{0}$ and $C_{0}$ are the inductance and capacitance at $x=0$. Using the TEM transmission line model (telegrapher's equations) and equations (2)-(4), the voltage $V(x)$ and current $I(x)$ in the frequency domain, along this line are defined as: 


$$
\left\{\begin{array}{l}
\frac{\partial^{2} V}{\partial x^{2}}-\frac{1}{Z(x)} \cdot \frac{\partial Z(x)}{\partial x} \cdot \frac{\partial V}{\partial x}+L \cdot C \cdot \omega^{2} \cdot V=0 \\
\frac{\partial^{2} I}{\partial^{2}}+\frac{1}{Z(x)} \cdot \frac{\partial \mathrm{Z}(\mathrm{x})}{\partial x} \cdot \frac{\partial}{\partial x}+L \cdot C \cdot \omega^{2} \cdot I=0
\end{array}\right.
$$

\subsection{Equations resolution}

The solution of the voltage equation is given by :

$$
\mathrm{V}(\mathrm{x})=\mathrm{D}(\mathrm{x}) \cdot \mathrm{p}+\mathrm{E}(\mathrm{x}) \cdot \mathrm{q}
$$

where $\mathrm{p}$ and $\mathrm{q}$ are two constants and $D(x), E(x)$ represent two power series expansions:

$$
D(x)=\sum_{j=0}^{\infty} d_{j} \cdot x^{j}
$$

and

$$
E(x)=\sum_{j=0}^{\infty} e_{j} \cdot x^{j+1}
$$

Then, the current relation is expressed by using the solution of the voltage (6) :

$$
I(x)=\frac{j \cdot v}{Z(x) \cdot \omega}\left(p \cdot D^{\prime}(x)+q \cdot E^{\prime}(x)\right)
$$

where $v$ is the propagation velocity through the line, $D^{\prime}(x)$ and $E^{\prime}(x)$ are the first derivatives of $D(x)$ and $E(x)$, respectively. The convergence of these four expansions is ensured by the Fuchs theorem. Two recurrence relations of order five are obtained by including (7) and (8) into (6) and then into (5), and by equating terms of same degrees : 


$$
\begin{aligned}
d_{j}=\{ & d_{j-1} \cdot Z_{1} \cdot \ell \cdot(j-1) \cdot(j-3)+ \\
& d_{j-2} \cdot\left[Z_{2} \cdot \ell^{2} \cdot(j-2) \cdot(j-5)+\beta^{2} \cdot Z_{0} \cdot \ell^{2}\right]+ \\
& d_{j-3}\left[Z_{3} \cdot \ell^{3} \cdot(j-3) \cdot(j-7)+\beta^{2} \cdot Z_{1} \cdot \ell^{3}\right]+ \\
& d_{j-4} \cdot \beta^{2} \cdot Z_{2} \cdot \ell^{4}+ \\
& \left.d_{j-5} \cdot \beta^{2} \cdot Z_{3} \cdot \ell^{5}\right\} /\left[-Z_{0} \cdot j \cdot(j-1)\right] \\
e_{j}= & e_{j-1} \cdot Z_{1} \cdot \ell \cdot j \cdot(j-2)+ \\
& e_{j-2} \cdot\left[Z_{2} \cdot \ell^{2} \cdot(j-1) \cdot(j-4)+\beta^{2} \cdot Z_{0} \cdot \ell^{2}\right]+ \\
& e_{j-3} \cdot\left[Z_{3} \cdot \ell^{3} \cdot(j-2) \cdot(j-6)+\beta^{2} \cdot Z_{1} \cdot \ell^{3}\right]+ \\
& e_{j-4} \cdot \beta^{2} \cdot Z_{2} \cdot \ell^{4}+ \\
& \left.e_{j-5} \cdot \beta^{2} \cdot Z_{3} \cdot \ell^{5}\right\} /\left[-Z_{0} \cdot j \cdot(j+1)\right]
\end{aligned}
$$

$$
\text { with } \quad \beta=\frac{\omega}{v}, \quad a_{0}=1 \quad \text { and } \quad b_{0}=\ell \text {. }
$$

\subsection{Scattering parameters of the whole line}

The figure 1 represents a line section between two discrete points, with $Z_{0}$ and $Z_{L}$, the characteristic impedance at the left- and right-hand sides, respectively.

Figure 1 : Line section and characteristic impedance

To determine the constants $p$ and $q$ in the $V(x)$ and $I(x)$ expressions, boundary conditions are applied. Thus, at the output end :

$$
\left.\frac{V(x)}{I(x)}\right|_{x=\ell}=Z_{L}
$$

where $Z_{L}=Z(x=\ell)$

This expression give the ratio : 


$$
\frac{p}{q}=-\frac{j \cdot E+\frac{v}{\omega} E^{\prime}}{j \cdot D+\frac{v}{\omega} D^{\prime}}
$$

with $D=D(x=\ell)$, and similarly for $E$, $D^{\prime}$ and $E^{\prime}$.

Then, at the input end we have :

$$
\left.\frac{V(x)}{I(x)}\right|_{x=0}=Z_{\text {in }}
$$

where $Z_{\text {in }}$ is the input impedance, which is expressed by using $p / q$. The associated reflection coefficient $\rho_{\text {in }}$ is defined as :

$$
1+\rho_{\text {in }}=\frac{2 Z_{\text {in }}}{Z_{\text {in }}+Z_{0}}
$$

The section line is terminated with a matching transmission line of characteristic impedance $Z_{L}$, so the scattering parameter $S_{21}(\omega)$ is given by :

$$
\mathrm{S}_{21}(\omega)=\sqrt{\frac{\mathrm{Z}_{0}}{\mathrm{Z}_{\mathrm{L}}}} \frac{\mathrm{V}(\mathrm{x}=\ell)}{\mathrm{V}(\mathrm{x}=0)}\left(1+\rho_{\text {in }}\right)
$$

Using this relation and the $p / q$ ratio, $S_{21}(\omega)$ is directly expressed from $D, E, D^{\prime}$ and $E^{\prime}$ :

$$
S_{21}(\omega)=\sqrt{\frac{Z_{0}}{Z_{L}}}(-2 j) \frac{D^{\prime} \cdot E-D \cdot E^{\prime}}{-\frac{\omega}{V} E+j \cdot E^{\prime}+j \cdot D+\frac{V}{\omega} \cdot D^{\prime}}
$$

At the input end, $\rho_{i n}$ is equal to the $S_{11}(\omega)$ scattering reflection coefficient :

$$
S_{11}(\omega)=\frac{-\frac{\omega}{v} E+j \cdot E^{\prime}-j \cdot D-\frac{v}{\omega} \cdot D^{\prime}}{-\frac{\omega}{v} E+j \cdot E^{\prime}+j \cdot D+\frac{v}{\omega} \cdot D^{\prime}}
$$

The reciprocity principle implies that $S_{12}(\omega)=S_{21}(\omega)$ and using the same approach, the parameter $S_{22}(\omega)$ is found as : 


$$
S_{22}(\omega)=\frac{-\frac{\omega}{v} E-j \cdot E^{\prime}+j \cdot D-\frac{v}{\omega} \cdot D^{\prime}}{-\frac{\omega}{v} E+j \cdot E^{\prime}+j \cdot D+\frac{v}{\omega} \cdot D^{\prime}}
$$

At this step, we have the knowledge of the four scattering parameters of a line section. But there are defined with impedance references $Z_{0}$ and $Z_{L}$. So changing-impedance reference formulas are used to pass at the common reference $50 \Omega$.

Concerning the analysis of the whole line, the same method is applied to the different line section. Then each scattering matrix is transformed in chain matrix, and then cascaded to be converted again into scattering parameters.

\subsection{Optimization of the whole line}

One of the main advantages of this method is the possibility to optimize the CVTL to achieve a microwave function, such as filtering for example. In optimization process, the freedom degrees of a system or device are in relation with the components of the optimization vector. Here, these components are the coordinates of the discrete points used in the cubic interpolation, e.g. $x_{j}$ positions and $Z_{j}$ impedances

For low-pass filters, the optimization was performed on the reflection coefficient $S_{11}(\omega)$. The algorithm is based on the Levenberg-Marquardt and J.J. Moré method [4], which uses the least-square criteria. The number of freedom degrees associated with several random generations of the input vector gives at the end of convergence process different transmission line shapes for responses that are in general equivalent. Finally, the choice of the best shape is determined by the more feasible profile.

To validate the theory, a stop-band and several low-pass filters have been designed and carried out in microstrip and coplanar technology [2][3]. A comparison with classical 
Chebyshev synthesis have shown differences in the 1-15 GHz range. Indeed, the losses in the band and the deviation between theory and measurement at the cut off frequency were higher for the Chebyshev than for the CVTL. Other filters were optimized to eliminate the spurious response of the $S_{21}$ parameter up to four times the cutoff frequency.

In telecommunication applications, band-pass filters are mostly used. So the next step is to extend this technique to these filters.

\section{APPLICATION FOR COUPLED TRANSMISSION LINES : BAND-PASS FILTERS}

For band-pass filters, we have chosen the topology of coupled transmission lines and applied the CVTL technique to each resonator. Because of the symmetry of the structure, the analysis is classically simplified by using the even and odd modes (figure 2) :

Figure 2 : Nonuniform coupled transmission lines

\subsection{Symmetry of the structure : even and odd modes}

Usually, only the coupling coefficient is taken into account [5], and the even impedance $Z_{0 e}(x)$ and odd impedance $Z_{0 o}(x)$ along the lines are deduced while keeping constant their product : $\sqrt{Z_{0 e}(x) \cdot Z_{0 o}(x)}=50 \Omega$. This constraint is particularly useful for couplers, but it brings restrictions for filters.

Moreover, the variations of even and odd mode impedance are both interpolated in the approach that we proposed, but separately. To design band-pass filters with coupled lines, the 4-port structure shown in Fig. 2 is converted into a 2-port quarter-wavelength resonator. So, using the notations of (7) and (8), $S_{21}$ and $S_{11}$ are given by : 
$S_{21}(\omega)=-j\left(\sqrt{\frac{Z_{0 e}}{Z_{L e}}} \cdot \frac{D_{e}^{\prime} \cdot E_{e}-D_{e} \cdot E_{e}^{\prime}}{-\frac{\omega}{v_{e}} \cdot E_{e}+j \cdot E_{e}^{\prime}+j \cdot D_{e}+\frac{v_{e}}{\omega} \cdot D_{e}^{\prime}}+\sqrt{\frac{Z_{0 o}}{Z_{L o}}} \cdot \frac{D_{o}^{\prime} \cdot E_{o}-D_{o} \cdot E_{o}^{\prime}}{-\frac{\omega}{v_{o}} \cdot E_{o}+j \cdot E_{o}^{\prime}+j \cdot D_{o}+\frac{v_{o}}{\omega} \cdot D_{o}^{\prime}}\right)$

$S_{11}(\omega)=\frac{1}{2}\left(\frac{-\frac{\omega}{v_{e}} \cdot E_{e}+j \cdot E_{e}^{\prime}-j \cdot D_{e}-\frac{v}{\omega} D_{e}^{\prime}}{-\frac{\omega}{v_{e}} \cdot E_{e}+j \cdot E^{\prime}{ }_{e}+j \cdot D_{e}+\frac{v}{\omega} \cdot D^{\prime}}+\frac{-\frac{\omega}{v_{o}} \cdot E_{o}+j \cdot E_{o}^{\prime}-j \cdot D_{o}-\frac{v}{\omega} D_{o}^{\prime}}{-\frac{\omega}{v_{o}} \cdot E_{o}+j \cdot E_{o_{o}}^{\prime}+j \cdot D_{o}+\frac{v}{\omega} \cdot D^{\prime}{ }_{o}}\right)$

where the index $e$ and $o$ are associated with the even and odd modes, respectively.

Before including open-end effects [6] and cascading these elements, we introduce in the proposed formalism the dispersion on even and odd characteristic impedance and effective permittivities. At each frequency step, the frequency-dependent values are calculated with accuracy [7] to evaluate the scattering parameters. The reference impedance that set the physical dimensions is chosen at the center frequency of the band-pass.

When resonators are cascaded, the continuity at the interconnection point (A plane on figure 3) is insured by using the same even and odd impedance values for both side of the structure.

Figure 3 : Configuration of two cascaded resonators

In both simulation and optimization, the even and odd impedance values are defined as two single continuously functions as shown in figure 4 . Note that the odd mode values must be always lower than even modes, which is guaranteed by constraints on the optimization process.

Figure 4 : Variations of odd and even impedance values 
Moreover as illustrated in figure 2, the bending out of the coupled line shape increases the line length $\left(l_{n u}>l_{u}\right)$ to be view by the waves. A simple integration method enable to evaluate the length $l_{n u}$ taken at the strip center.

In the next step, the different resonators are cascaded by using the chain matrix following the design of the coupled lines. The number of resonators depends on the filter order.

Finally, nonuniform single lines are connected to the filter ends to provide smooth transitions (Figure 3), while of course being simulated.

\subsection{Optimization of the two modes to eliminate spurious responses}

Other methods to design nonuniform band-pass filters don't take into account the two different phase velocities. These ones implies a spurious band-pass at twice the center frequency of the desired band-pass. Thus, a compensation method to eliminate this phase velocity difference has to be added. A saw-tooth or serpentine modification of the inner edges coupled lines is widely used [5].

Here, a posteriori phase velocity compensation is not required while a complete analysis of odd and even mode is achieved. In fact, the originality of the method is based on odd and even impedance nonuniform variations to generate low-pass or stop-band characteristic in the pass-band structure. Another technique allows the coupled lines to overlap outside the resonator proper [8]. This configuration makes the odd mode length longer then the even mode and thus compensates for the phase velocity difference. So, the parasitic band-pass is largely removes.

\subsection{1 : First order filter - first spurious response elimination}


The method capabilities are first tested to minimize the effects of the phase-velocity difference due to the inhomogeneous propagation medium of microstrip coupled lines. Indeed, the aim is to get better band-pass symmetry and lower parasitic at twice the center frequency than with common coupled-line microstrip filters. Two simulation results are presented; the first one is the $S_{21}$ simulation parameter for the uniform coupled lines, and the second one the simulation of the optimized nonuniform filter. Experimental results are given for validation (Fig. 5-b). Four discrete points per quarter-wavelength elements are sufficient to describe this filter impedance variation (see Fig. 5 -a) designed on alumina substrate $\left(\varepsilon_{\mathrm{r}}=\right.$ 9.6, $\mathrm{h}=0.635 \mathrm{~mm}$, thickness metallization : $t=5 \mu \mathrm{m}$, synthesis frequency : $5 \mathrm{GHz}$ ). The degrees of freedom allows us to control the spurious responses of the $S_{21}$ parameter while keeping the same band-pass response.

Figure 5 : Order one filter with compensated velocity

(a) Microstrip profile - (b) Frequency responses.

experimental results for the nonuniform lines
theory for the nonuniform lines
theory for the uniform lines

\subsection{2 : Third order filter}

To validate the technique, filter of higher order have been designed and realized. The goal was to obtain better rejection and to reject spurious response of $S_{21}$ parameter on larger band-pass. The figures 6,7 and 8 give the profile of the third order filter, the transmission parameter and the return losses : 
Figure 6 : Profile of the third order filter

Figure 7 : Transmission parameter of the third order filter

Figure 8 : Return losses of the third order filter

It can be noted a good agreement in the band-pass but a slight discrepancy near 10 GHz. This is probably due to the lack of precise model for the open lines.

\section{CONCLUSION.}

A new method for band-pass filters design has been proposed. Firstly, the technique using CVTL was developed for low pass filters. Based on a simple mathematical formalism without approximation (in the frame of TEM mode), the method generates a line shape which permits the optimization of the filter characteristics. It has been shown a very good agreement with measures over a large range of frequencies $(0-50 \mathrm{GHz})$ [3]. Then, the technique has been extended to band-pass filters through the coupled lines topology. Analysis and optimization was performed separately on the even and odd modes of the symmetric structure to ensure the most complete description. So, in a first step, the natural different phase velocity of the microstrip technology was compensated by using the nonuniform shape of the different coupled lines. Then higher order filter was designed and realized to obtain better rejection and eliminate spurious band-passes of the transmission parameter. Nevertheless, coupled lines are one of the many topologies for band-pass filters. So, it will be interesting to applied the CVTL at other structures to obtain better performances, particularly at millimeter frequencies. Indeed, at these frequencies, the absence of discontinuities should introduce no radiation and show the interest of the concept. 


\section{References}

1. Gupta K. C. : “Emerging trends in millimeter-wave CAD”, IEEE MTT, vol. 46, n6, June 1998, pp 747,755.

2. M. Le Roy, A. Pérennec, L.C. Calvez, S. Toutain, “A new design of microwave filters by using continuously varying transmission lines,” IEEE Microwave Theory and Tech. Symposium, pp. 639-642, 1997.

3., M. Le Roy, A. Pérennec, L.C. Calvez, S. Toutain “ The continuously varying transmission lines technique - Application to filter design ”, IEEE MTT,vol. 47, $n^{\circ}$ 9, part I, September 1999, pp. 1680, 1687

4. J.J. Moré, “The Levenberg-Marquardt algorithm : implementation and theory,” Lecture notes in mathematics 630, 1978, Springer Verlag.

5. S. UysaL, A.H. Aghvami, "Synthesis, design, and construction of ultra-wide-band nonuniform quadrature directional couplers in inhomogeneous media," IEEE Trans. Microwave Theory and Tech, vol. 37, pp. 969-975, June 1989.

6. M. Kirschning, R.H. Jansen, N.H. Koster, “Accurate model for open end effect of microstrip lines,” Electron. Lett., vol. 17, n³, pp 123-125, 1981 . 
7. M. Kirschning, R.H. Jansen, “Accurate wide-range design equations for the frequencydependent characteristics of parallel coupled microstrip lines,” IEEE Trans. Microwave Theory and Tech., vol. 32, pp. 83-90, 1984.

8. A. Riddle "High performance parallel coupled microstrip filters” IEEE MTT Symposium, New York, 25 - 27 may, pp 427, 430, 1988 


\section{Figures Captions}

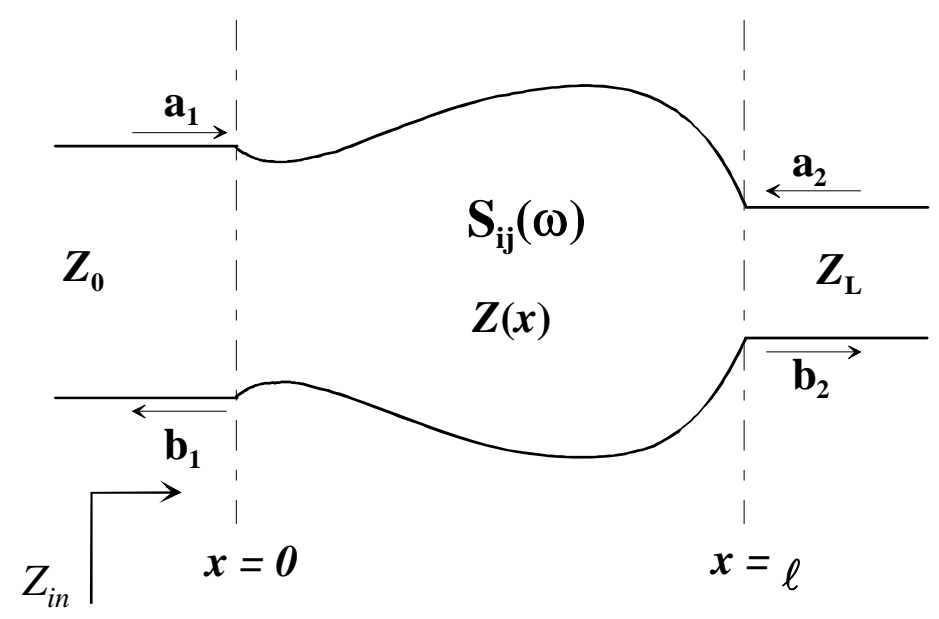

Figure 1 : Line section and characteristic impedance

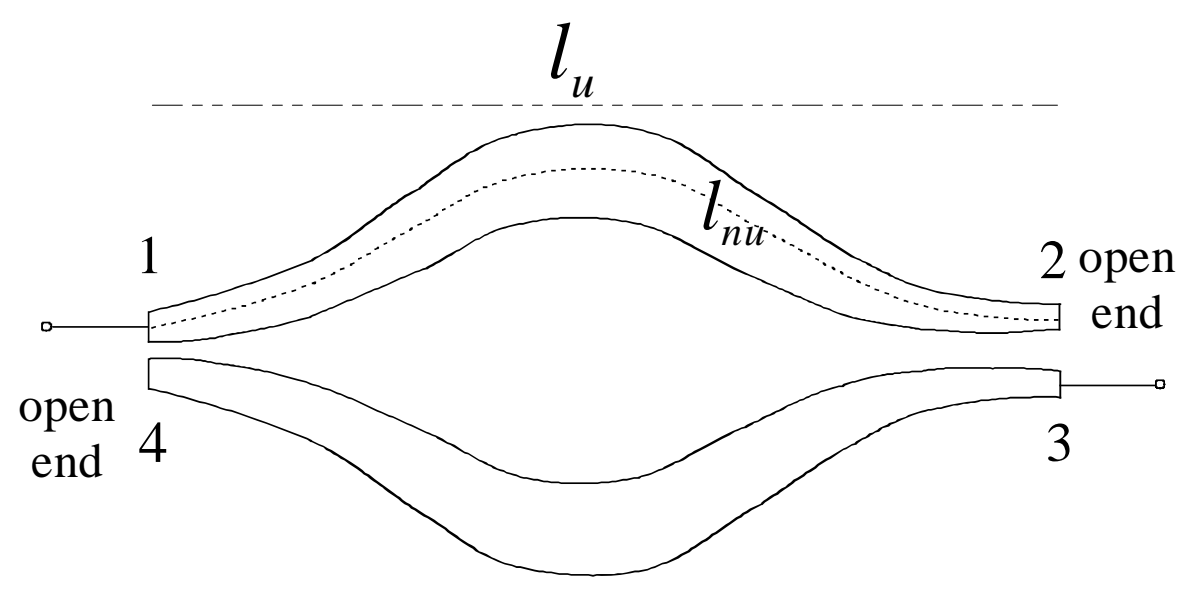

Figure 2 : Nonuniform coupled transmission lines

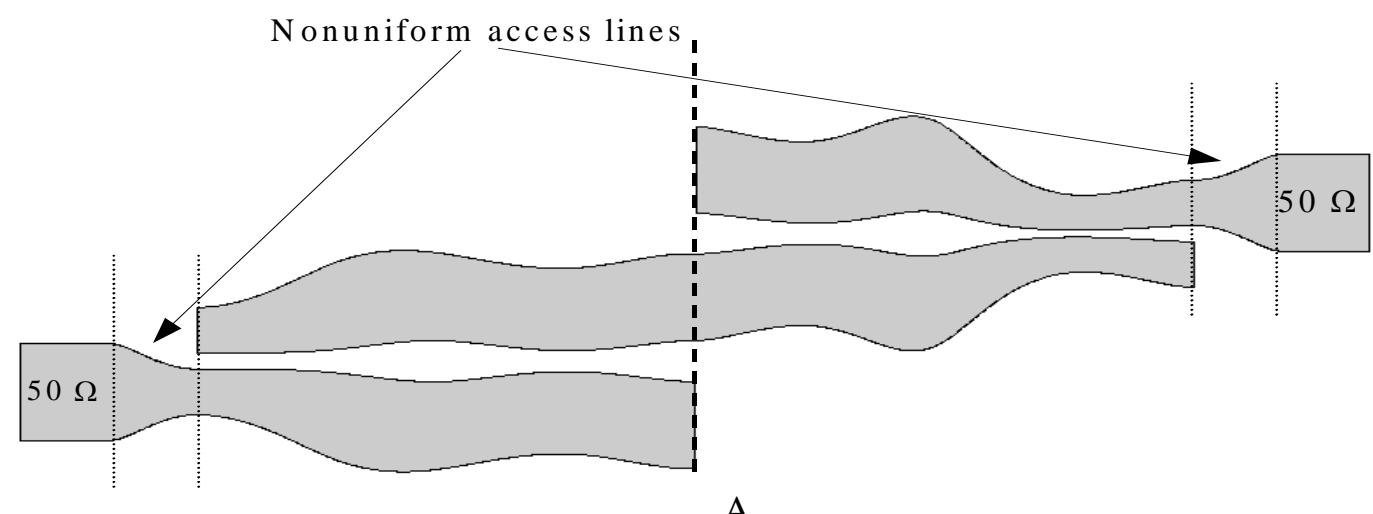

A

Figure 3 : Configuration of two cascaded resonators 


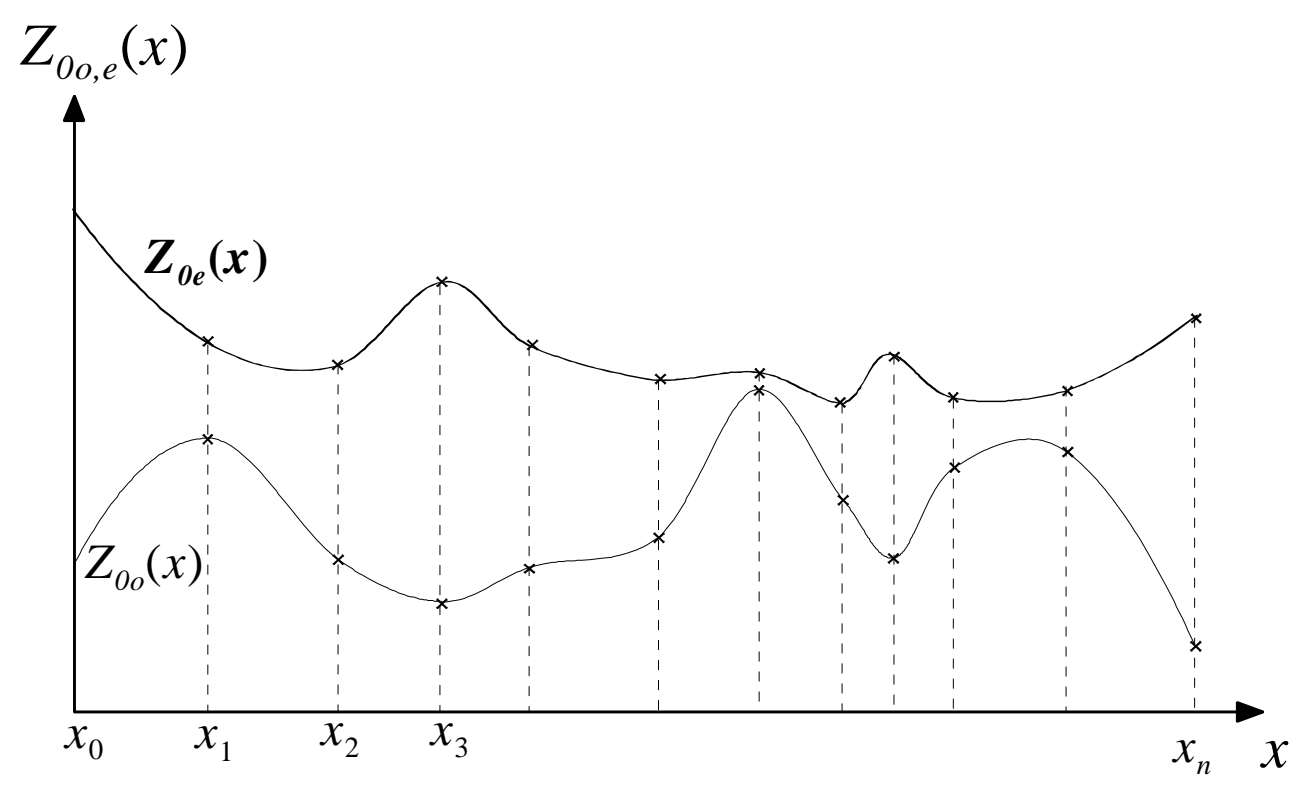

Figure 4 : Variations of odd and even impedance values

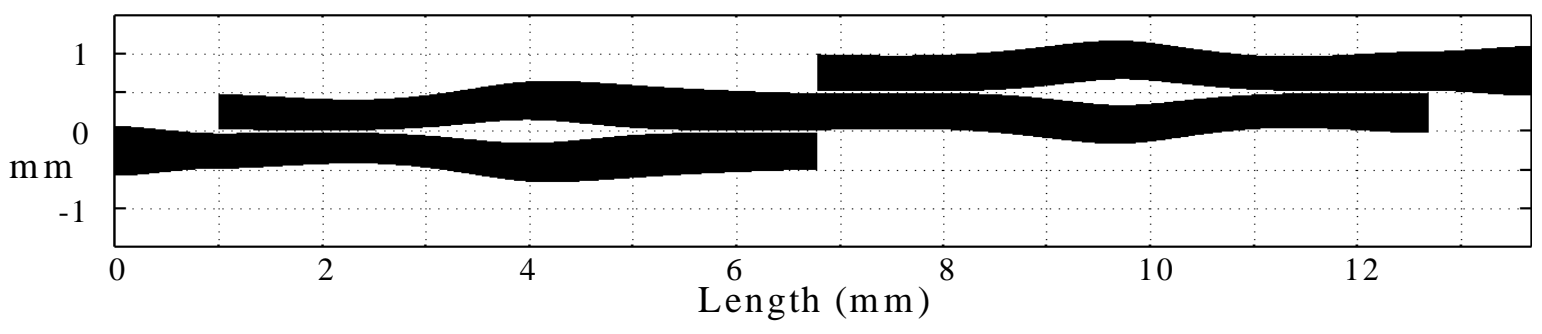

(a)

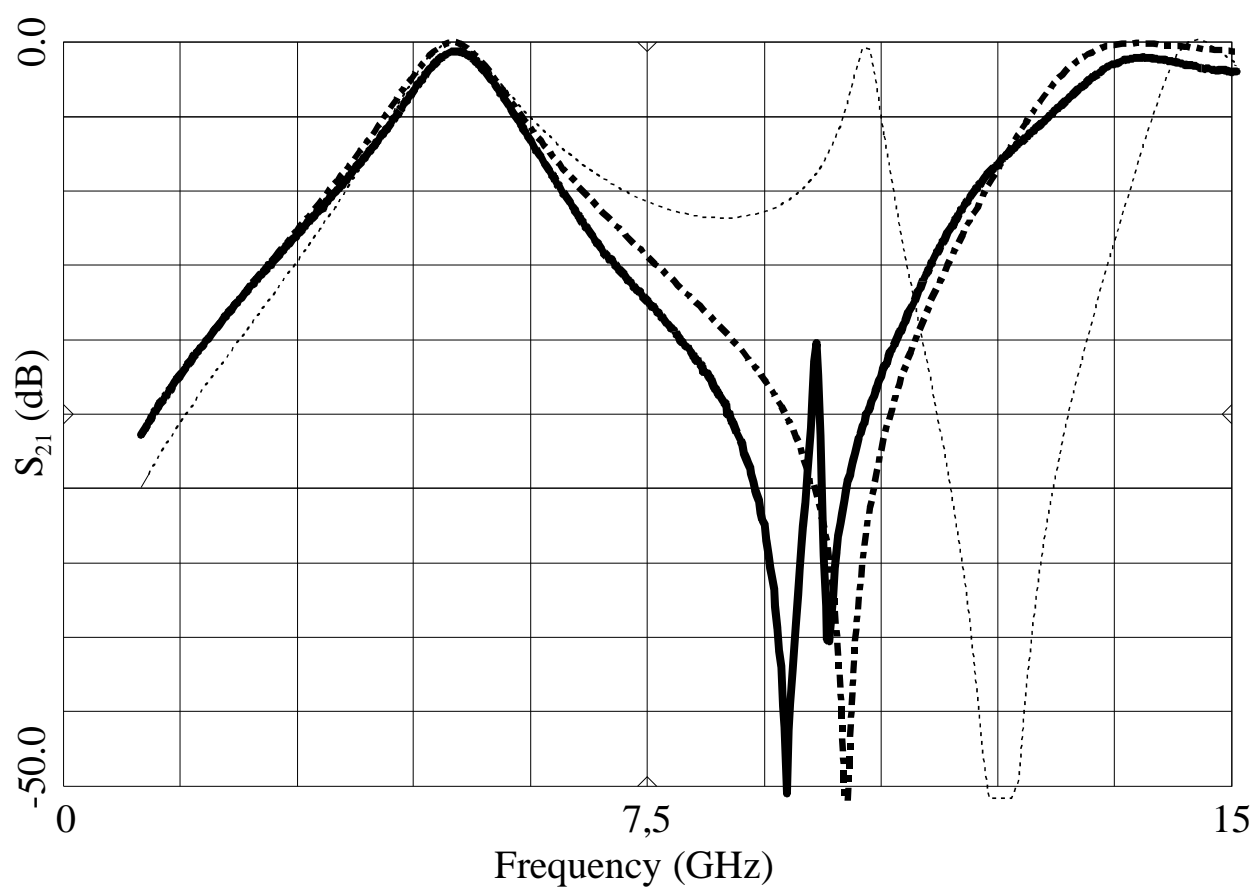

(b) 
Figure 5 : Order one filter with compensated velocity

(a) Microstrip profile - (b) Frequency responses.
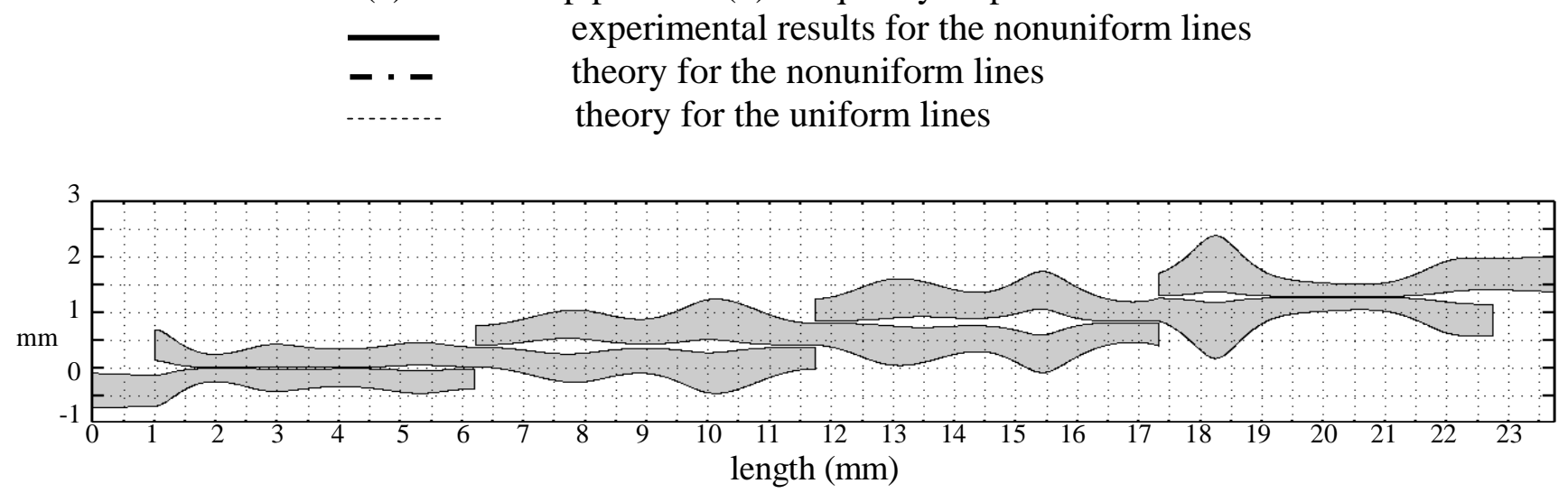

Figure 6 : Profile of the third order filter

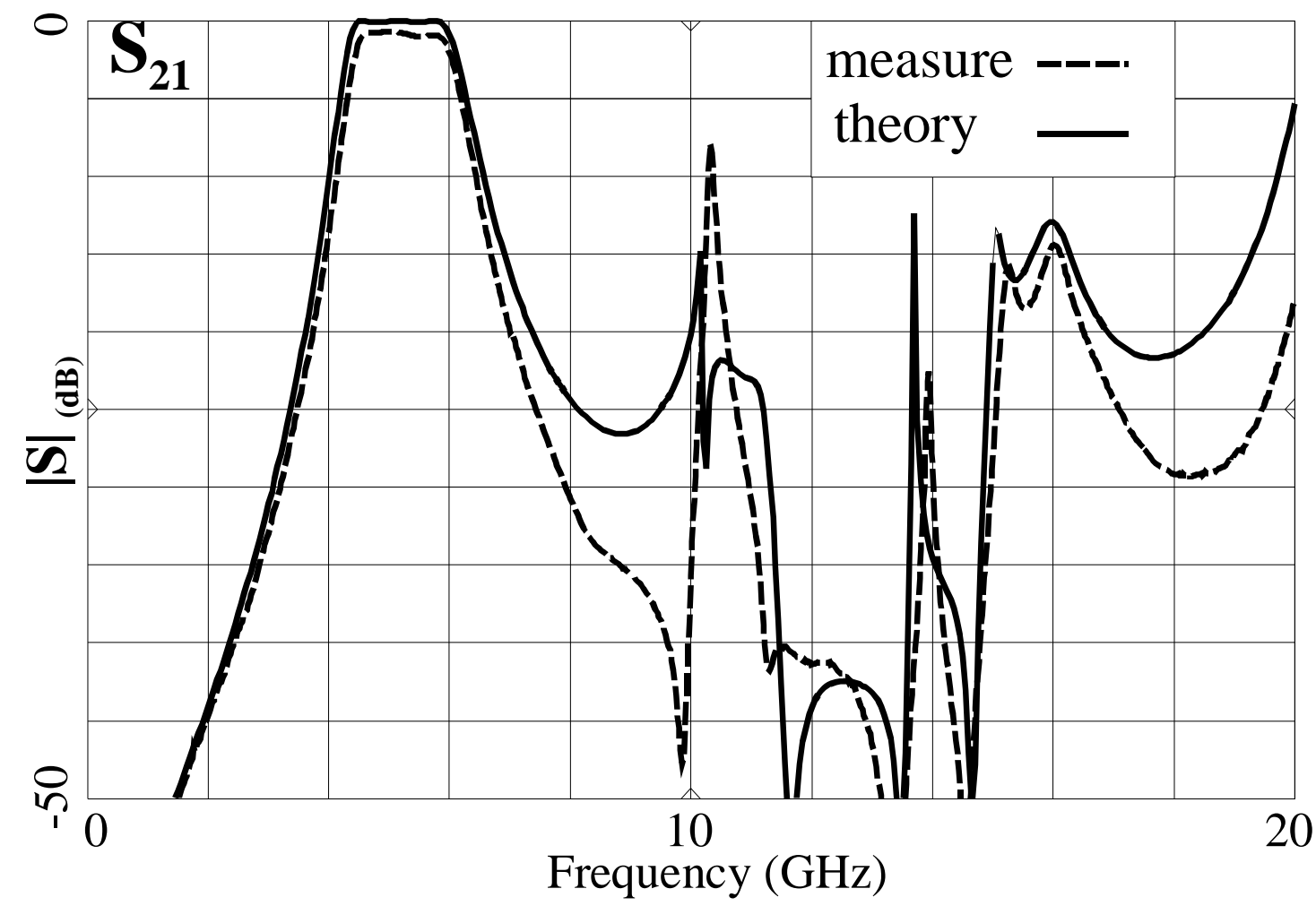

Figure 7 : Transmission parameter of the third order filter 


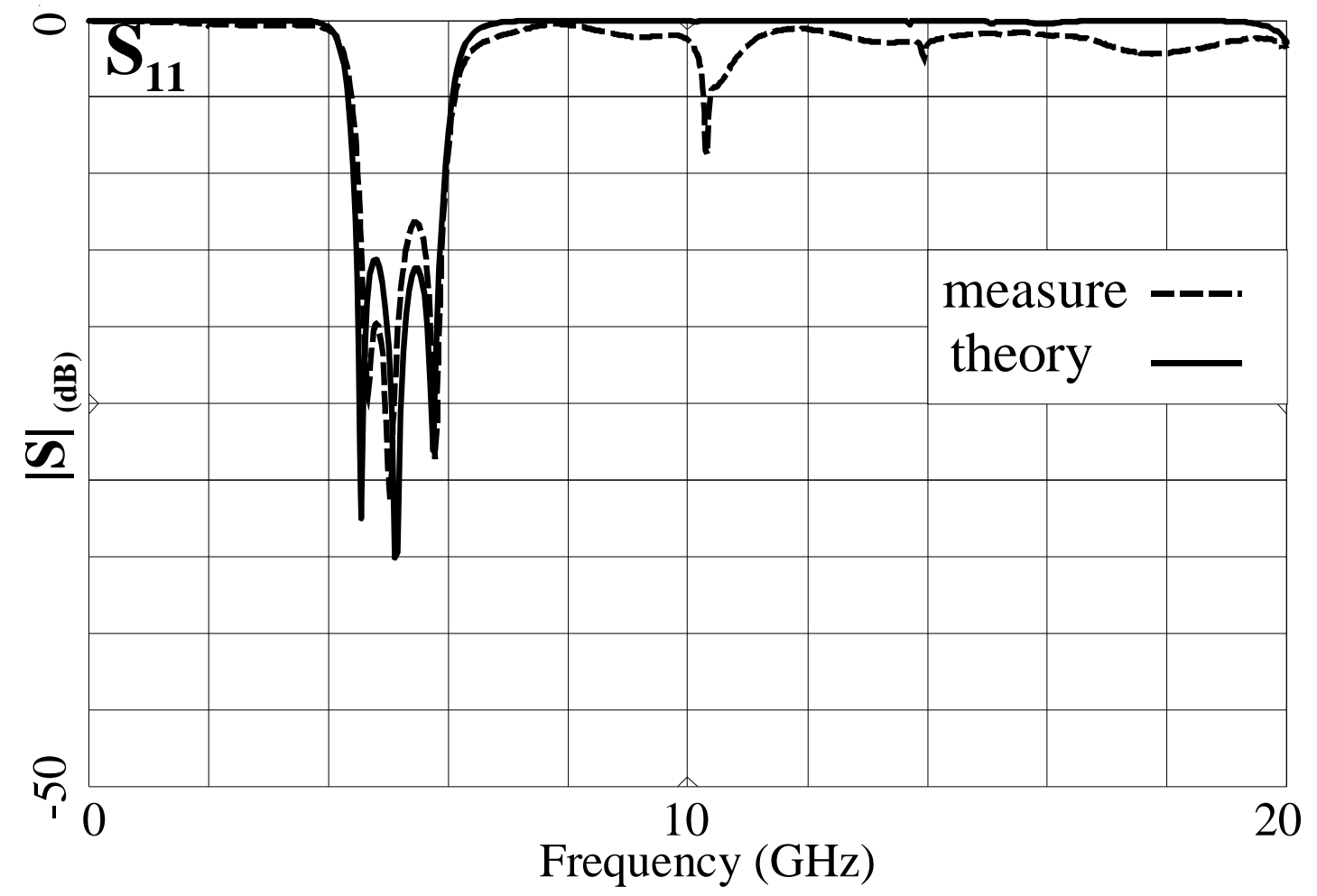

Figure 8 : Return losses of the third order filter 
Keywords:

Nonuniform Transmission Lines, band-pass filters, optimization

Abbreviated title

Nonuniform coupled filter 